\title{
Dynamical systems with benign ghosts
}

\author{
Thibault Damour $\oplus^{*}$ \\ Institut des Hautes Etudes Scientifiques, 91440 Bures-sur-Yvette, France \\ Andrei Smilga $\oplus^{\dagger}$ \\ SUBATECH, Université de Nantes, 4 rue Alfred Kastler, BP 20722, Nantes 44307, France
}

(Received 18 November 2021; accepted 8 February 2022; published 23 February 2022)

\begin{abstract}
We consider finite and infinite-dimensional ghost-ridden dynamical systems whose Hamiltonians involve nonpositive definite kinetic terms. We point out the existence of three classes of such systems where the ghosts are benign, i.e., systems whose evolution is unlimited in time: (i) systems obtained from the variation of bounded-motion systems; (ii) systems describing motions over certain Lorentzian manifolds and (iii) higher-derivative models related to certain modified Korteweg-de Vries equations.
\end{abstract}

DOI: 10.1103/PhysRevD.105.045018

\section{INTRODUCTION}

A ghost-ridden dynamical quantum system is defined as a system whose spectrum is not bounded neither from below, nor from above. This is in particular the case for the quantum versions of the Ostrogradsky Hamiltonians [1], describing the dynamics of higher-derivative Lagrangians (for a review, see, e.g., [2]). Many ghost-ridden systems are sick: their evolution operator is not unitary. Such systems involve classical trajectories that run into a singularity after a finite time of evolution (a blowup).

There are, however, ghost-ridden systems with benign ghosts, in the sense that they do not exhibit a classical blowup and have a unitary quantum evolution operator. One of the simplest examples of such a benign-ghost system is the PaisUhlenbeck oscillator [3] with the higher-derivative Lagrangian,

$$
L=\frac{1}{2}\left[\ddot{x}^{2}-\left(\omega_{1}^{2}+\omega_{2}^{2}\right) \dot{x}^{2}+\omega_{1}^{2} \omega_{2}^{2} x^{2}\right] .
$$

The quantum version of the corresponding Ostrogradsky Hamiltonian does not have a bottom. Indeed, there exists a canonical transformation $[4,5]$ that brings the Hamiltonian into the form, ${ }^{1}$

\footnotetext{
*damour@ihes.fr

†smilga@subatech.in2p3.fr

${ }^{1}$ We assumed here that $\omega_{1} \neq \omega_{2}$ (and took $\omega_{1}>\omega_{2}>0$ ). In the case of equal frequencies, the situation is somewhat more complicated because the canonical transformation mentioned above is singular and the Hamiltonian is not reduced to the form (1.2). Still, the Hamiltonian is well defined. It has a continuous spectrum $[3,6,7]$.

Published by the American Physical Society under the terms of the Creative Commons Attribution 4.0 International license. Further distribution of this work must maintain attribution to the author(s) and the published article's title, journal citation, and DOI. Funded by SCOAP.
}

$$
H=\frac{\hat{P}_{1}^{2}+\omega_{1}^{2} X_{1}^{2}}{2}-\frac{\hat{P}_{2}^{2}+\omega_{2}^{2} X_{2}^{2}}{2} .
$$

This Hamiltonian has a pure point spectrum with neither a bottom, nor a ceiling,

$$
E_{n m}=\left(n+\frac{1}{2}\right) \omega_{1}-\left(m+\frac{1}{2}\right) \omega_{2} .
$$

If the ratio $\omega_{1} / \omega_{2}$ is irrational, the spectrum is dense everywhere. The evolution operator is unitary.

The ghosts generally strike back, however, when one leaves the realm of quadratic Hamiltonians to include interactions. Consider, for example, the Lagrangian,

$$
L=\frac{1}{2}\left[\ddot{x}^{2}-2 \omega^{2} \dot{x}^{2}+\omega^{4} x^{2}\right]-\frac{1}{4} \alpha x^{4},
$$

whose classical equation of motion reads

$$
\left(\frac{d^{2}}{d t^{2}}+\omega^{2}\right)^{2} x-\alpha x^{3}=0 .
$$

The classical trajectories depend on four initial conditions. There is an obvious stationary point,

$$
x(0)=\dot{x}(0)=\ddot{x}(0)=x^{(3)}(0)=0 .
$$

The behavior of the system in the vicinity of this point depends on the sign of $\alpha$. If $\alpha>0$, the point (1.6) lies within an "island of stability"- the trajectories with initial conditions sufficiently close to (1.6) do not go astray but exhibit an oscillatory behavior. However, this island has a shore. When the deviations of initial conditions from (1.6) are large enough, the trajectory blows up [8]. 
If $\alpha<0$, the island of stability for the system (1.5) shrinks to the single point (1.6). All the trajectories except $x(t)=0$ run away to infinity in a finite time. This does not happen, however, for the Pais-Uhlenbeck oscillator with unequal frequencies.

In most cases, an island of stability with a finite phasespace volume is present. For instance, a similar island of stability (surrounded by an infinite ocean of blowup behavior) was observed in [9] for a (cosmology-inspired) model obtained by adding to the Pais-Uhlenbeck Hamiltonian (1.2) (with $\omega_{1}=0$ and $\omega_{2} \neq 0$ ) an interaction term $\lambda X_{1}^{2} X_{2}^{2}$ (with $\lambda>0$ ). A proof of the existence of an island of stability for (nonresonant) Pais-Uhlenbeck Hamiltonians perturbed by a general interaction term $\mu_{3}\left(X_{1}-X_{2}\right)^{3}+\mu_{4}\left(X_{1}-X_{2}\right)^{4}+O\left[\left(X_{1}-X_{2}\right)^{5}\right]$ was given in Ref. [4].

When considering the quantum version of ghost-ridden Hamiltonians involving nontrivial interactions, it is difficult to reach definite answers applying to general situations. A case by case study is required. For instance, it has been argued that the quantum problem for the system (1.4) is also malignant [2]. On the other hand, there are Hamiltonians which entail blowing up classical motions but which lead to a well-defined, unitary quantum evolution. A well-known example is the Hamiltonian describing the three-dimensional motion of a particle in an attractive $\frac{1}{r^{2}}$ potential,

$$
H=\frac{\vec{p}^{2}}{2 m}-\frac{\kappa}{r^{2}} .
$$

Classically, for certain initial conditions, the particle falls to the center in a finite time. The quantum dynamics of this system depends on the value of $\kappa$. If $m \kappa<1 / 8$, the ground state exists, and unitarity is preserved. If $m \kappa>1 / 8$, the spectrum is not bounded from below and, what is worse, the quantum problem cannot be well posed until the singularity at the origin is smoothed out [10]. One can say that for $m \kappa<1 / 8$, quantum fluctuations cope successfully with the attractive force of the potential and prevent the system from collapsing.

The latter example suggests that quantum fluctuations can only make a ghost-ridden system better, not worse. We therefore conjecture that, if the classical dynamics of the system is benign, its quantum dynamics will also be benign, irrespective of whether the spectrum has, or does not have, a bottom.

Some particular examples of benign ghost-ridden (nonlinearly interacting) Hamiltonians have been presented in previous works $[8,11-15]$. The aim of the present paper is to delineate new classes of benign Hamiltonians, some of which have a large generality (in the sense that they contain several arbitrary functions). More precisely, we shall present classes of nontrivially interacting ghost-ridden systems such that all the classical motions (and not only the motions restricted to a limited region of phase space) admit an infinite-time evolution. ${ }^{2}$ In view of the conjecture stated in the last paragraph, we expect that the quantum dynamics of our benign ghost-ridden systems will be well posed. We leave studies of this issue to future work.

\section{VARIATIONAL DYNAMICS: A LARGE CLASS OF BENIGN GHOSTS}

A general class of dynamical systems with benign ghosts is obtained by considering the variational equations of motion of a bounded-motion ${ }^{3}$ Hamiltonian system. An example of such a system was found in [11] by studying a certain higher-derivative supersymmetric mechanical system, though its variational nature was not noticed. $^{4}$

The general setting for defining such systems is the following. One starts from a basic, unperturbed dynamical system (with $n$ degrees of freedom) described, say, by a Lagrangian, $L_{0}\left(q^{i}, \dot{q}^{i}\right), i=1, \cdots n$, i.e., by the action,

$$
S_{0}[q, \dot{q}]=\int d t L_{0}\left(q^{i}, \dot{q}^{i}\right) .
$$

Then, one considers the dynamics defined by varying the Lagrangian action (2.1), i.e., by making in $L_{0}\left(q^{i}, \dot{q}^{i}\right)$ the replacement,

$$
\begin{aligned}
q^{i}(t) & \rightarrow q^{i}(t)+\epsilon Q^{i}(t), \\
\dot{q}^{i}(t) & \rightarrow \dot{q}^{i}(t)+\epsilon \dot{Q}^{i}(t),
\end{aligned}
$$

and by keeping only the term linear in $\epsilon$. In other words, one is considering the new action (with $2 n$ degrees of freedom),

$$
S_{1}[q, \dot{q} ; Q, \dot{Q}]=\int d t L_{1}\left(q^{i}, \dot{q}^{i} ; Q^{i}, \dot{Q}^{i}\right)
$$

where

$L_{1}\left(q^{i}, \dot{q}^{i} ; Q^{i}, \dot{Q}^{i}\right)=Q^{j} \frac{\partial L_{0}(q, \dot{q})}{\partial q^{j}}+\dot{Q}^{j} \frac{\partial L_{0}(q, \dot{q})}{\partial \dot{q}^{j}}$.

The variational action (2.3) leads to the following equations of motion for $q^{i}$ and $Q^{i}$ (with $\delta / \delta q$ denoting an EulerLagrange variational derivative, $\left.\partial_{q}-\frac{d}{d t} \partial_{\dot{q}}+\frac{d^{2}}{d t^{2}} \partial_{\ddot{q}}+\cdots\right)$ :

\footnotetext{
${ }^{2}$ Note that we are not requiring that the motions indefinitely stay within a compact region of phase space. We do not exclude power law, or exponential, runaway behaviors. We are simply excluding finite-time blowup.

${ }^{3}$ Here, "bounded motion" means that the time evolution of the considered, unperturbed, system stays within some compact domain of phase space.

${ }^{4}$ The supersymmetric aspects of this problem are not relevant here, and we shall forget here about fermions.
} 


$$
\begin{aligned}
& 0=\frac{\delta L_{1}}{\delta Q^{i}}=\frac{\partial L_{0}(q, \dot{q})}{\partial q^{i}}-\frac{d}{d t} \frac{\partial L_{0}(q, \dot{q})}{\partial \dot{q}^{i}} \equiv \frac{\delta L_{0}}{\delta q^{i}}, \\
& 0=\frac{\delta L_{1}}{\delta q^{i}}=Q^{j} \frac{\partial L_{0}(q, \dot{q})}{\partial q^{j} \partial q^{i}}+\dot{Q}^{j} \frac{\partial L_{0}(q, \dot{q})}{\partial \dot{q}^{j} \partial q^{i}}-\frac{d}{d t}\left[Q^{j} \frac{\partial L_{0}(q, \dot{q})}{\partial q^{j} \partial \dot{q}^{i}}\right]-\frac{d}{d t}\left[\dot{Q}^{j} \frac{\partial L_{0}(q, \dot{q})}{\partial \dot{q}^{j} \partial \dot{q}^{i}}\right] .
\end{aligned}
$$

We see that the first Euler-Lagrange equation $0=\delta L_{1} / \delta Q^{i}$ coincides with the unperturbed equation of motion of $q^{i}$, $\delta L_{0}(q, \dot{q}) / \delta q^{i}=0$. On the other hand, the second equation, $0=\delta L_{1} / \delta q^{i}$ coincides with the variation of the equation $\delta L_{0}(q, \dot{q}) / \delta q^{i}=0$ : it is obtained by replacing there $q^{i}(t)$ and their first and second derivatives as in (2.2) and keeping only the term linear in $\epsilon$.

When considering the first-order Hamiltonian version of the so-defined variational dynamics, one encounters a slightly surprising feature. Namely, if we denote the momenta conjugate to $q^{i}$ and $Q^{i}$, respectively, as $p_{i}$ and $P_{i}$, the Hamiltonian $H_{1}\left(q^{i}, Q^{i} ; p_{i}, P_{i}\right)$ describing the varied dynamics [corresponding to $L_{1}\left(q^{i}, \dot{q}^{i} ; Q^{i}, \dot{Q}^{i}\right)$ ] is not obtained by varying the unperturbed Hamiltonian $H_{0}\left(q^{i} ; p_{i}\right)$ by means of the naively expected variation $q^{i} \rightarrow q^{i}+\epsilon Q^{i} ; p_{i} \rightarrow p_{i}+\epsilon P_{i}$. It is obtained by first doing the latter naive variation and then by swapping the momenta according to $p_{i} \leftrightarrow P_{i}$.

The necessity to swap $p \leftrightarrow P$ in the naive variation of $H_{0}\left(q^{i} ; p_{i}\right)$ is easily seen by varying the Hamiltonian version of the action (2.1),

$$
S_{0}^{H}[q, p]=\int d t\left[p_{i} \dot{q}^{i}-H_{0}(q, p)\right] .
$$

Using the naively defined variations $q^{i} \rightarrow q^{i}+\epsilon Q^{i}$; $p_{i} \rightarrow p_{i}+\epsilon P_{i}$, we get

$$
\begin{aligned}
\delta S_{0}^{H}[q, Q, p, P] & =\int d t\left[P_{i} \dot{q}^{i}+p_{i} \dot{Q}^{i}\right. \\
& \left.-\left(Q^{i} \frac{\partial H_{0}(q, p)}{\partial q^{i}}+P_{i} \frac{\partial H_{0}(q, p)}{\partial p_{i}}\right)\right],
\end{aligned}
$$

in which the Hamiltonian kinetic term $P_{i} \dot{q}^{i}+p_{i} \dot{Q}^{i}$ shows that the conjugate momentum to $q^{i}$ is actually $P_{i} \sim \delta p_{i}$, while the conjugate momentum to $Q^{i}$ is the unperturbed $p_{i}$. After the swap $p \leftrightarrow P$, one finally gets the Hamiltonian for the varied dynamics expressed in terms of the canonical pairs $(q, p) ;(Q, P)$,

$H_{1}(q, Q ; p, P)=Q^{i} \frac{\partial H_{0}(q, P)}{\partial q^{i}}+p_{i} \frac{\partial H_{0}(q, P)}{\partial P_{i}}$.

From our present perspective (namely, studying ghostridden dynamics), note that all the varied Hamiltonians $H_{1}(q, Q ; p, P)$ are necessarily unbounded below (and above) because they have a linear dependence on the phase-space variables $Q^{i}$ and $p_{i}$; see Eq. (2.8). This makes the quantum spectrum also unbounded. Let us, however, see why this ghost feature leads generically to a benign evolution.

The simplest type of varied dynamics is obtained by varying a simple Hamiltonian (with 1 degree of freedom) of the form,

$$
H_{0}(x, p)=\frac{1}{2} p^{2}+V(x)
$$

In that case, the variation of the first term yields the symmetric structure $p P$, invariant under the swap $p \leftrightarrow P$, which is thus ineffective. One then gets (with the notation $D \equiv Q=\delta x$ used in [11]) a varied-dynamics Hamiltonian involving two pairs of canonically conjugated variables, $(x, p)$ and $(D, P)$,

$$
H_{1}(x, D ; p, P)=p P+D V^{\prime}(x) .
$$

The classical equations of motion are

$$
\ddot{x}+V^{\prime}(x)=0, \quad \ddot{D}+V^{\prime \prime}(x) D=0 .
$$

They admit two constants of motion: the varied Hamiltonian $H_{1}(x, D ; p, P)$ and the conserved energy $E_{0}=\frac{1}{2} \dot{x}^{2}+V(x)$ of the unperturbed motion of $x$. Using the Hamilton equation $\dot{x}=\frac{\partial H_{1}}{\partial p}=P$, the unperturbed energy $E_{0}(x, \dot{x})$ yields the second integral of motion $N(x, P)$ with

$$
N(x, P)=\frac{P^{2}}{2}+V(x)
$$

The model of Ref. [11] was of this type, with the simplest nontrivial potential,

$$
V(x)=\frac{\omega^{2} x^{2}}{2}+\frac{\lambda x^{4}}{4}, \quad \lambda>0 .
$$

If one introduces the variables,

$$
X_{1,2}=\sqrt{\frac{\omega}{2}} x \pm \frac{1}{\sqrt{2 \omega}} D, \quad P_{1,2}=\frac{1}{\sqrt{2 \omega}} p \pm \sqrt{\frac{\omega}{2}} P,
$$

the Hamiltonian (2.10) acquires the form [11], 
$H_{1}=\frac{P_{1}^{2}+\omega^{2} X_{1}^{2}}{2}-\frac{P_{2}^{2}+\omega^{2} X_{2}^{2}}{2}+\frac{\lambda}{4 \omega}\left(X_{1}-X_{2}\right)\left(X_{1}+X_{2}\right)^{3}$.

In other words, this is the Hamiltonian (1.2) with degenerate frequencies, with an additional quartic interaction of a special form.

A nice distinguishing feature of the system (2.10) is its integrability. Indeed, we have 2 degrees of freedom and two commuting integrals of motion: $H_{1}$ and $N$. (The vanishing of the Poisson bracket $\left\{H_{1}, N\right\}$ is easily checked.) This allows one to find, for the simple potential (2.13), the solution analytically.

First, the unperturbed dynamics for $x$ describes oscillations in the quartic potential (2.13). The solutions are elliptic functions whose parameters depend on the integral of motion $N$,

$$
x(t)=x_{0} \operatorname{cn}\left[\Omega\left(t-t_{0}\right), k\right],
$$

where $\mathrm{cn}$ is a Jacobi elliptic function (with elliptic modulus $k=\sqrt{m}$ ), and

$$
\begin{aligned}
\alpha & =\frac{\omega^{4}}{\lambda N}, \quad \Omega=[\lambda N(4+\alpha)]^{1 / 4} \\
k^{2} \equiv m & =\frac{1}{2}\left[1-\sqrt{\frac{\alpha}{4+\alpha}}\right], \quad x_{0}=\left(\frac{N}{\lambda}\right)^{1 / 4} \sqrt{\sqrt{4+\alpha}-\sqrt{\alpha}}
\end{aligned}
$$

Bearing in mind (2.16), the equation for $D$ is a Hill equation describing an oscillator with periodically varying frequency. The solutions of generic Hill equations are obtained by Floquet theory and, depending on parameters, can be either bounded for all times, or exponentially growing. However, we are here in a special case. We know that the equation for $D$ describes the infinitesimal variations of the general solution of the quartic oscillator describing the $x$ dynamics. Therefore, we can simply obtain the general solution for $D$ by varying the integration constants entering the general solution (2.16). It is enough to get two independent solutions. A first one can be obtained by varying the integration constant $t_{0}$ in Eq. (2.16). This yields

$D_{1}(t)=\frac{\partial[x(t)]}{\partial t_{0}}=-\dot{x}(t)=+\Omega x_{0} \operatorname{sn}[\Omega t, k] \operatorname{dn}[\Omega t, k]$,

where we have set $t_{0}=0$ after variation. This solution is periodic in $t$.

A second solution is obtained by varying the value of $N$ in Eq. (2.16). Varying $N$ implies that both the amplitude $x_{0}(N)$, and the frequency $\Omega(N)$, of $x(t)$ must be varied. The variation of $\Omega(N)$ in $x(t)=x_{0}(N) \operatorname{cn}[\Omega(N) t, k]$ (setting again $t_{0}=0$, and noticing that $k$ does not depend on $N$ ) brings about a prefactor $t$ multiplying the derivative of $\mathrm{cn}(x)$ and generates a $D_{2}(t)$ of the form,

$$
\begin{aligned}
D_{2}(t) & =t \frac{d}{d t} \operatorname{cn}[\Omega(N) t, k]+\beta \operatorname{cn}[\Omega(N) t, k] \\
& =-t \Omega(N) \operatorname{sn}[\Omega(N) t, k] \operatorname{dn}[\Omega(N) t, k]+\beta \operatorname{cn}[\Omega(N) t, k],
\end{aligned}
$$

where $\beta$ is some constant. The contribution $-t \Omega \operatorname{sn}[\Omega t, k] \operatorname{dn}[\Omega t, k]$ is the product of $t$ by a periodic function of $t$. Therefore, while the first independent solution $D_{1}(t)$ is periodic, the second independent solution $D_{2}(t)$ will exhibit an oscillatory behavior with an amplitude rising linearly in time. This is illustrated in Fig. 1. What is important for our present purpose is the benign nature of the general solution, $D(t)=c_{1} D_{1}(t)+c_{2} D_{2}(t)$. Indeed, we consider as malignant ghost only the cases leading to blowup in a finite time. The linear growth in time exhibited by the generic solution of the $D(t)$ equation is quite benign.

We refer to Ref. [11] for a study of the quantum version of the Hamiltonian (2.10) (due to integrability, the eigenvalues, and eigenfunctions of the quantum Hamiltonian can be found explicitly in this case).

We have used here the specific quartic potential (2.13) to be able to exhibit explicit solutions of the equations of motion for the system (2.10). However, the conclusions we reached about the benign dynamics of the $(x, D ; p, P)$ system hold for a general class of potentials. Indeed, if we take for $V(x)$, a smooth confining potential growing as $x \rightarrow \pm \infty$, the solutions for $x(t)$ will represent a nonlinear oscillation of a certain type-periodic functions of time with a frequency $\Omega$ depending on the integral of motion,

$$
E_{0}(x, \dot{x}) \equiv \frac{1}{2} \dot{x}^{2}+V(x)=\frac{P^{2}}{2}+V(x) \equiv N .
$$

In other words,

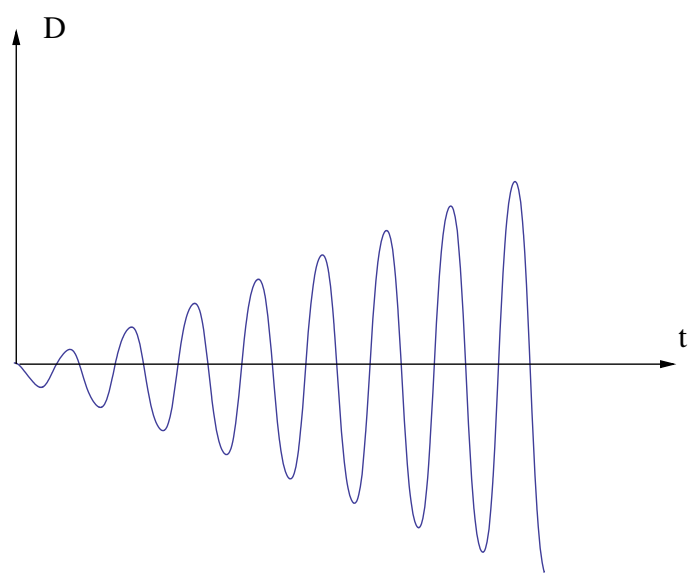

FIG. 1. A typical behavior of $D(t)$, as follows from solving Eq. (2.11). 


$$
x(t)=X\left[\Omega(N)\left(t-t_{0}\right), N\right],
$$

where $X(\theta, N)$ is a periodic function of $\theta$, with period $2 \pi$. It can be expanded into a Fourier series,

$$
X(\theta, N)=\operatorname{Re}\left\{\sum_{k \in \mathbb{Z}} A_{k}(N) e^{\mathrm{i} k \theta}\right\} .
$$

The existence of the two commuting integrals of motion $H_{1}$ and $N$ allows one to solve the motion of $x(t)$ by a quadrature, giving some type of hyperelliptic function.

The general solution for $D(t)$ can then be simply obtained by varying the general solution for $x(t)$ with respect to the two integration constants it contains, namely $t_{0}$ and $N$. Again, it is clear that the first independent solution,

$D_{1}(t)=\partial x(t) / \partial t_{0}=-\dot{x}(t)=\Omega(N) \operatorname{Im}\left\{\sum_{k} k A_{k}(N) e^{\mathrm{i} k \theta(t)}\right\}$,

will be periodic in time [with the same period as $x(t)$ ], while the second solution,

$$
\begin{aligned}
D_{2}(t) & =\frac{\partial}{\partial N} X\left[\Omega(N)\left(t-t_{0}\right), N\right] \\
& =\operatorname{Re} \frac{\partial}{\partial N}\left\{\sum_{k} A_{k}(N) e^{\mathrm{i} k \Omega(N)\left(t-t_{0}\right)}\right\},
\end{aligned}
$$

will be the sum of a periodic function, and of the function,

$$
\frac{d \ln \Omega(N)}{d N}\left(t-t_{0}\right) \dot{x}(t) .
$$

The latter function is a product of $t-t_{0}$ and of a periodic function of $t$. As in the case illustrated in Fig. 1, we have again an oscillatory behavior with an amplitude rising linearly in time. A benign ghost again.

Let us consider the generalization of these results to $n$ degrees of freedom, i.e., to the system with the varied Lagrangian (2.4) and the corresponding Hamiltonian (2.8). In that case, the results will strongly depend on the integrable or nonintegrable character of the unperturbed dynamics described by the action (2.1), or equivalently, (2.6).

Let us first assume that the unperturbed dynamics (2.6) is integrable and that we are considering bounded motions. In that case, the general solution of the dynamics (2.6) corresponds to a quasiperiodic motion, where the phasespace coordinates $q^{i}, p_{i}$ admit representations of the form,
$q^{i}(t)=q^{i}[\mathbf{I}, \boldsymbol{\theta}(t)]=\sum_{\mathbf{k}} a_{k_{1} \cdots k_{n}}^{i}\left(I_{1}, \ldots, I_{n}\right) e^{\mathrm{i}\left(k_{1} \theta_{1}(t)+\cdots+k_{n} \theta_{n}(t)\right)}$,

$p_{i}(t)=p_{i}[\mathbf{I}, \boldsymbol{\theta}(t)]=\sum_{\mathbf{k}} b_{k_{1} \cdots k_{n}}^{i}\left(I_{1}, \ldots, I_{n}\right) e^{\mathrm{i}\left(k_{1} \theta_{1}(t)+\cdots+k_{n} \theta_{n}(t)\right)}$.

Here, $(\mathbf{I}, \boldsymbol{\theta})=\left(I_{i}, \theta_{i}\right), i=1, \ldots, n$ are action-angle variables, $\mathbf{k}=\left(k_{i}\right), i=1, \ldots, n$ are multiplets of (relative) integers (summed over $\mathbb{Z}^{n}$ ), and the time evolution of the angles is of the form,

$$
\theta_{i}(t)=\omega_{i}(\mathbf{I}) t+\theta_{i}^{0}
$$

We assume that the $2 n$ integration constants entering this solution are $I_{i}$, and $\theta_{i}^{0}$. The general solution for the variation $Q^{i}=\delta q^{i}$ can then be obtained as a linear superposition of the $2 n$ particular varied solutions defined by varying the $2 n$ integration constants, i.e.,

$$
Q^{i}(t)=\sum_{j}\left[C_{I_{j}} \frac{\partial}{\partial I_{j}} q^{i}[\mathbf{I}, \boldsymbol{\theta}(t)]+C_{\theta_{j}} \frac{\partial}{\partial \theta_{j}^{0}} q^{i}[\mathbf{I}, \boldsymbol{\theta}(t)]\right] .
$$

This expression is the sum of some quasiperiodic functions [of the type (2.26)] and of the functions coming from varying the frequencies $\omega_{i}\left(I_{j}\right)$, namely,

$$
t \sum_{j, \ell} C_{I_{j}} \frac{\partial \omega_{\ell}}{\partial I_{j}} \frac{\partial q^{i}[\mathbf{I}, \boldsymbol{\theta}]}{\partial \theta_{\ell}} .
$$

The latter functions are the products of $t$ and quasiperiodic functions of time. ${ }^{5}$ This is again a benign behavior of linearly growing oscillatory form, which is essentially a quasiperiodic version of Fig. 1.

Let us now consider the generic case where the unperturbed system (2.1) is not integrable and exhibits a chaotic behavior. [We are still assuming that the (unperturbed) evolution stays within a compact domain of phase space.] In that case, the behavior of the varied dynamical system can be much worse than in the integrable case assumed above. First, on midterm time scales, the variations $Q^{i}=\delta q^{i}$ may grow exponentially with time (Lyapunov instability). On longer time scales, such a Lyapunov exponential instability might (via an Arnold-type diffusion) evolve into a more chaotic behavior. Anyway, $Q^{i}(t)$ satisfies an homogeneous linear ordinary differential equation (ODE) with time-dependent coefficients, say (in the simple, equal-mass, potential case)

\footnotetext{
${ }^{5}$ Here, we assume a fast enough decay for the coefficients $a_{k_{1} \ldots k_{n}}^{i}$ as the $k_{i}$ tend to infinity to ensure the quasiperiodic nature of the right factor in Eq. (2.29).
} 


$$
\ddot{Q}^{i}+\frac{\partial^{2} V(q)}{\partial q^{i} \partial q^{j}} Q^{j}=0
$$

in which $q^{i}$ must be replaced by a solution of $\ddot{q}^{i}+\frac{\partial V(q)}{\partial q^{i}}=0$. General theorems about linear ODE's then guarantee that $Q^{i}(t)$ can only have singularities at a finite time if the coefficients of the ODE become singular. As we assume here that the unperturbed solution $q^{i}(t)$ is regular for all times, we are guaranteed that the behavior of $Q^{i}(t)$ will be benign in our general sense (i.e., no finite-time blowup).

Let us finally mention that our variational approach can be straightforwardly generalized to field theory systems (i.e., to an infinite number of dynamical variables). For instance, if we start with the unperturbed Lagrangian [using here the signature $\left.\left(x_{\mu}\right)^{2} \equiv t^{2}-\mathbf{x}^{2}\right]$,

$$
\mathcal{L}_{0}=\frac{1}{2}\left(\partial_{\mu} \phi\right)^{2}-V(\phi),
$$

we are led to the following variational Lagrangian for two real fields $\phi(t, \mathbf{x})$ and $D(t, \mathbf{x}) \equiv \delta \phi(t, \mathbf{x})$ :

$$
\mathcal{L}=\partial_{\mu} \phi \partial_{\mu} D-D V^{\prime}(\phi) .
$$

If we use the same potential as in (2.10), the equations of motion for the system (2.32) are

$$
\begin{aligned}
\square \phi+\omega^{2} \phi+\lambda \phi^{3} & =0, \\
\square D+\left(\omega^{2}+3 \lambda \phi^{2}\right) D & =0 .
\end{aligned}
$$

The particular case of a $(1+1)$-dimensional spacetime has been considered (and numerically investigated) in Ref. [14].

General mathematical results on the nonlinear KleinGordon equation (with potential $\lambda \phi^{p} / p$, with $\lambda>0$ and $p<2+\frac{4}{d-2}$ in $d$ spatial dimensions) [16] guarantee the global existence of solutions of Eq. (2.33) for $\phi(t, \mathbf{x})$, with suitable (finite-energy) Cauchy data at $t=0$. These generic solutions have been shown to exhibit asymptotic decay in time. We then expect that these exact results on the nonlinear Klein-Gordon equation for $\phi$ imply (by varying the Cauchy data) a benign behavior (with asymptotic decay) for generic solutions of the linear, varied KleinGordon equation satisfied by $D=\delta \phi$. Reference [14] numerically investigated the case of a one-dimensional space $(d=1)$ compactified on a circle. The space compactification suppresses the asymptotic time decay of $\phi$ and seemingly induced a mild (linear in time) growth for $D$ [14].

Evidently, our variational construction can be set up basically for any system. One can vary the Yang-Mills Lagrangian,

$$
\mathcal{L}_{0}^{\mathrm{YM}}=-\frac{1}{2} \operatorname{Tr}\left\{F_{\mu \nu} F^{\mu \nu}\right\}
$$

setting $A_{\mu} \rightarrow A_{\mu}+\epsilon B_{\mu}$ and keeping in (2.34) the terms linear in $B_{\mu}$, namely,

$$
\mathcal{L}_{1}^{\mathrm{YM}}=B_{\mu} \frac{\delta \mathcal{L}^{\mathrm{YM}}[A]}{\delta A_{\mu}} .
$$

At the linearized level (in $A$ ), $\mathcal{L}_{1}^{\mathrm{YM}}$ describes two massless spin-1 fields, one of which is a ghost. At the nonlinear level, the number of degrees of freedom is preserved because of the invariance of $\mathcal{L}_{1}^{\mathrm{YM}}$ under two distinct gauge transformations: the usual one acting on $A$, and a separate one (involving $A$-covariant derivatives) acting on $B$. This system is again a benign ghost-ridden system.

Mutatis mutandis, one can also consider the ghost-ridden action obtained by varying the Einstein action [we use here the signature $(-+++)$ and $16 \pi G=1]$,

$$
\mathcal{L}_{0}^{\mathrm{E}}=\sqrt{-g} g^{\mu \nu} R_{\mu \nu}[g] .
$$

Varying $g_{\mu \nu} \rightarrow g_{\mu \nu}+\epsilon h_{\mu \nu}$ and keeping the term linear in $\epsilon$ yields

$$
\mathcal{L}_{1}^{\mathrm{E}}=-\sqrt{-g}\left(R^{\mu \nu}[g]-\frac{1}{2} g^{\mu \nu} R[g]\right) h_{\mu \nu} .
$$

At the linearized level around flat spacetime $\left(g_{\mu \nu}=\right.$ $\left.\eta_{\mu \nu}+\epsilon f_{\mu \nu}\right), \mathcal{L}_{1}^{\mathrm{E}}$ describes two massless spin-2 fields, one of which is a ghost. At the nonlinear level, the number of degrees of freedom is preserved because of the invariance of $\mathcal{L}_{1}^{\mathrm{E}}$ under two distinct gauge transformations: the usual coordinate transformations, $x^{\prime \mu}=f^{\mu}\left(x^{\nu}\right)$, acting both on $g_{\mu \nu}$ and on the tensor $h_{\mu \nu}$, and a separate one (involving $g$-covariant derivatives) acting linearly on $h$, namely $h_{\mu \nu} \rightarrow h_{\mu \nu}+\nabla_{\mu}^{g} \xi_{\nu}+\nabla_{\nu}^{g} \xi_{\mu}$. Note that the Euler-Lagrange equations derived from $\mathcal{L}_{1}^{\mathrm{E}}$ imply that $g_{\mu \nu}$ must satisfy the (vacuum) Einstein equations,

$$
\mathcal{E}^{\mu \nu}\left[g_{\alpha \beta}\right] \equiv \sqrt{-g}\left(R^{\mu \nu}[g]-\frac{1}{2} g^{\mu \nu} R[g]\right)=0,
$$

while $h_{\mu \nu}$ must satisfy the linearized Einstein equations,

$$
\frac{\delta \mathcal{E}^{\mu \nu}}{\delta g_{\alpha \beta}} h_{\alpha \beta}=0 .
$$

This system is again a benign ghost-ridden system. For instance, the mathematical results on the global nonlinear stability of the Minkowski spacetime [17-19] have shown the global existence of solutions of Einstein vacuum equations for $g_{\mu \nu}(t, \mathbf{x})$, with small Cauchy data at $t=0$. These generic solutions have been shown to exhibit 
asymptotic decay in time. We believe that the good control (in all spacetime directions) of the geometric properties of these nonlinear, but small perturbations of Minkowski space [17] suffice to prove the global existence of the Green's function needed to solve the linearized Einstein equation satisfied by $h_{\mu \nu}$ (after a suitable gauge fixing).

The basic reason why the ghost-ridden systems considered in this section were benign is that the equations of motion of the variables $(Q, P)$ were linear [though influenced by the nonlinear dynamics of the unperturbed variables $(q, p)]$. In the following, we are going to introduce more interesting ghost-ridden systems where the dynamics of the ghost degrees of freedom is nonlinear. It is then more delicate to delineate ghost-ridden systems that stay benign in spite of such nonlinear interactions.

\section{GEODESICS ON LORENTZIAN MANIFOLDS}

A general class of nonlinear ghost-ridden Hamiltonians with benign solutions are the (quadratic) Hamiltonians describing geodesic motion on geodesically complete Lorentzian manifolds. Namely, to any given $D$-dimensional Lorentzian manifold $M_{D}$ with metric tensor $g_{\mu \nu}(x)$, one can associate the Hamiltonian,

$$
H\left(x^{\lambda}, p_{\lambda}\right)=\frac{1}{2} g^{\mu \nu}(x) p_{\mu} p_{\nu} .
$$

Because of the $-+\cdots+$ signature that we choose to use here, this Hamiltonian contains $D-1$ positive terms and a ghostlike negative term. The Hamiltonian (3.1) describes geodesic motions on $M_{D}$. More precisely, the Hamilton equations of motion,

$$
\begin{aligned}
& \frac{d x^{\mu}}{d \tau}=\frac{\partial H}{\partial p_{\mu}}=g^{\mu \nu}(x) p_{\nu}, \\
& \frac{d p_{\mu}}{d \tau}=-\frac{\partial H}{\partial x^{\mu}}=-\frac{1}{2} \partial_{\mu} g^{\nu \lambda}(x) p_{\nu} p_{\lambda},
\end{aligned}
$$

describe a motion in the 2D-dimensional phase space $\left(x^{\lambda}, p_{\lambda}\right)$ [the cotangent space of $M_{D}$ ] with respect to an Hamiltonian "time variable" $\tau$, which is an affine parameter along the considered geodesic. For a general curved spacetime, the only conserved quantity of the dynamics (3.2) is the "energy" (the factor 2 being introduced for convenience),

$$
E=2 H(x, p) \equiv g^{\mu \nu}(x) p_{\mu} p_{\nu} .
$$

A positive value of $E$ describes spacelike geodesics, a negative value describes timelike ones, while $E=0$ describes null geodesics. Actually, as the affine parametrization of geodesics is defined modulo an arbitrary affine transformation $\tau \rightarrow a \tau+b$, one can, without loss of generality only consider the three cases: $E=+1, E=-1$, and $E=0$. The values $E= \pm 1$ mean that $\tau$ is equal to the proper length $\sqrt{ \pm d s^{2}}$ along the geodesic, with

$$
d s^{2}=g_{\mu \nu}(x) d x^{\mu} d x^{\nu}
$$

We are interested in the systems for which the Hamiltonian evolution (3.2) can be continued indefinitely with respect to the Hamiltonian time variable $\tau$. Then, as was argued in the Introduction, the ghosts are benign. In the context of the geodesic Hamiltonian (3.1), our condition boils down to saying that the Lorentzian manifold $\left(M_{D}, g\right)$ is geodesically complete. We can therefore conclude that the ghost-ridden Hamiltonian (3.1) defines a benign dynamics (for all values of the energy $E$ ) on any geodesically complete Lorentzian manifold.

Mathematical investigations have given large classes of geodesically complete Lorentzian manifolds. Of particular physical significance is the fact, proven in Refs. [20,21], that the vacuum Einstein spacetimes close to Minkowski that were constructed in Refs. [17-19] are geodesically complete for all values of the energy. Evidently, this property will not extend if one considers spacetimes containing black holes.

As a very particular type of geodesically complete spacetimes, we can also mention the de Sitter, as well as anti-de Sitter (AdS), spacetimes (of any dimension) [22]. For concreteness, let us consider the AdS spacetime, with the metric (in global coordinates),

$$
d s^{2}=-\left(1+\frac{r^{2}}{\ell^{2}}\right) d t^{2}+\frac{d r^{2}}{1+\frac{r^{2}}{\ell^{2}}}+r^{2} d \Omega_{D-2}^{2} .
$$

The associated geodesic Hamiltonian reads

$$
H=-\frac{1}{2} \frac{p_{t}^{2}}{1+\frac{r^{2}}{\ell^{2}}}+\frac{1}{2}\left(1+\frac{r^{2}}{\ell^{2}}\right) p_{r}^{2}+\frac{1}{2} \frac{J^{2}}{r^{2}}
$$

Here, $\ell$ is the AdS length scale giving the constant negative curvature $K=-1 / \ell^{2}$ and $J^{2}$ is the squared angular momentum linked to the motion on the sphere $S_{D-2}$.

The AdS spacetime is a homogeneous, symmetric space, equivalent to the coset $O(2, D-1) / O(1, D-1)$. Because of its homogeneity we can reduce the study of geodesics to the geodesics starting from any given point, say the origin $t=0, r=0$ in the global coordinates of (3.5). In addition, as the isotropy group of the coset $O(2, D-1) /$ $O(1, D-1)$, namely $O(1, D-1)$ is the local Lorentz group, we can use this group to reduce the study of the three types of geodesics to a particular timelike geodesic (e.g., the geodesic $r=0$ ), a particular spacelike one (e.g., the radial geodesic $t=0, \Omega=\mathrm{cst}$ ), and a particular null geodesic (a radial one directed along the light cone). 
In these cases, the solutions to the Eqs. (3.2) are very simple ${ }^{6}$ : the spacelike geodesic with $E=1$ is $r(\tau)=\sinh \tau$, $t=0, \Omega=\mathrm{cst}$, the timelike geodesic with $E=-1$ is $r=0, t=\tau, \Omega=\mathrm{cst}$, while the null geodesic is $r=\tau$, $t=\arctan \tau, \Omega=$ cst. We see that all three types of geodesics have an infinite affine length.

The class of Ricci-flat, near-Minkowski geodesically complete Lorentzian manifolds mentioned above [20,21] might naively suggest that any Lorentzian manifold with small enough curvature will be geodesically complete (as is the case for Riemannian manifolds). In other words, one might think that the incompleteness of geodesics must be linked to the presence of curvature singularities or at least of high-curvature regions. This is incorrect. The (toy-TaubNUT) Misner manifold [23] (see also Sec. V.8 in [22]) yields a simple example of a smooth Lorentzian manifold, which is locally flat, but geodesically incomplete (because of its nontrivial topology). The Misner manifold is two dimensional and has the topology $\mathbb{R} \times S_{1}$. In global coordinates $(x, \phi)$, where $x \in \mathbb{R}$ and where $\phi \in[0,2 \pi]$ is an angle describing the circle $S_{1}$, the metric reads ${ }^{7}$

$$
d s^{2}=2 d x d \phi+x d \phi^{2}
$$

This corresponds to the geodesic Hamiltonian (multiplied by a factor of 2 compared to Eq. (3.1)),

$$
H\left(x, \phi ; p_{x}, p_{\phi}\right)=2 p_{x} p_{\phi}-x p_{x}^{2} .
$$

Note that the metric and the Hamiltonian stay regular as one crosses the line $x=0$ (which is a Killing horizon). The determinant of the metric is everywhere equal to -1 , and the signature is globally -+ , so that the Hamiltonian is not positive definite, representing locally a difference between two squares. In other words, it involves an ordinary and a ghost degree of freedom.

The equations of motion read

$$
\begin{aligned}
\dot{x} & =2 p_{\phi}-2 x p_{x}, \\
\dot{\phi} & =2 p_{x}, \\
\dot{p}_{x} & =p_{x}^{2}, \\
\dot{p}_{\phi} & =0 .
\end{aligned}
$$

Denoting here the Hamiltonian time variable as $t$ (i.e., $\dot{x}=\frac{d x}{d t}$ ), it is simple to solve the equations of motion. We see that $p_{\phi}$ is an integral of motion. Assuming that the initial value of $p_{x}$ is not zero, say $p_{x}(0)=1 / c$, the equation of motion for $p_{x}$ is easily integrated, giving

\footnotetext{
${ }^{6}$ To simplify them still further, we have set $\ell=1$.

${ }^{7}$ See [22] for the local coordinate transformation needed to exhibit the flatness of the Misner metric (3.7).
}

$$
p_{x}(t)=\frac{1}{c-t} .
$$

This leads to a blowup for $p_{x}(t)$ at the finite time $t=c$, which can be positive or negative. Correspondingly, $\phi(t)$ blows up logarithmically as $t \rightarrow c$ according to

$$
\phi(t)=\phi(0)-2 \ln \left(1-\frac{t}{c}\right)
$$

assuming $t / c<1$. While $p_{\phi}$ stays constant, the time evolution of the remaining phase space variable $x(t)$ is conveniently obtained by using the constancy of the energy: $E=H\left(x, \phi ; p_{x}, p_{\phi}\right)$. This yields

$$
x=\frac{2 p_{x} p_{\phi}-E}{p_{x}^{2}} .
$$

When one approaches the blowup (where $p_{x} \rightarrow \infty$ ), $x$ tends to zero.

One can visualize the Misner manifold as a cylinder (with $\phi$ being the angular coordinate and $x$ the coordinate along its axis). The geodesic incompleteness of the Misner manifold means that most geodesics [apart from the ones with $p_{x}(0)=0$ ] spiral [either for $t<0$ or $t>0$, depending on the sign of $\left.p_{x}(0)\right]$ towards the horizon circle $x=0$ by making infinitely many turns within a finite (affine) time. This exemplifies how the nonlinearity of the ghost-ridden Hamiltonian (3.8) leads to a finite-time blowup in phase space, i.e., (3.8) is a system with a malignant ghost.

\section{MODIFIED KORTEWEG-DE VRIES EQUATION AS A BENIGN HIGHER-DERIVATIVE MODEL}

We started our discussion with the Pais-Uhlenbeck oscillator (1.1), a higher-derivative model with benign ghosts. We noted in the Introduction that the ghosts generically cease to be benign if a nonlinear interaction term is added to the Lagrangian (1.1). The ghost models considered in Sec. II were benign essentially because the ghost degrees of freedom satisfied linear equations of motion. The ghost models considered in Sec. III involved nonlinear evolution equations, and could be benign (or not) depending on the global geometric properties of the considered Lorentzian manifold. However, they were not models linked to higher-derivative Lagrangians.

A natural question at this stage is are there nonlinear higher-derivative models with benign ghosts? In Ref. [24], one of us suggested that the usual two-dimensional $(t, x)$ Korteweg-de Vries (KdV) system, with the roles of temporal and spatial variables interchanged might be benign because of the existence of infinitely many local conservation laws. Indeed, let us rename

$$
t \rightarrow X, \quad x \rightarrow T
$$


Then the local flux conservations, $0=\partial_{t} J_{n}^{t}+\partial_{x} J_{n}^{x} \equiv$ $\partial_{T} J_{n}^{x}+\partial_{X} J_{n}^{t}$, imply the $T$ conservation of the fluxes,

$$
\mathcal{F}_{n}=\int d X\left[J_{n}^{x}\right]_{T=\mathrm{cst}} \equiv \int d t\left[J_{n}^{x}\right]_{x=\mathrm{cst}} .
$$

Let us recall that the ordinary $\mathrm{KdV}$ equation for $u(t, x)$, namely, ${ }^{8}$

$$
u_{x x x}+6 u u_{x}+u_{t}=0
$$

derives from the action $\int d t d x L[\psi(t, x)]$ with the twodimensional Lagrangian density,

$$
L[\psi(t, x)]=\frac{1}{2} \psi_{x x}^{2}-\psi_{x}^{3}-\frac{1}{2} \psi_{t} \psi_{x},
$$

if one denotes $u(t, x) \equiv \psi_{x}$ after having varied over $\psi(t, x)$.

When changing the names of spacetime variables according to Eq. (4.1) and denoting $\psi(t, x)=\psi(X, T) \rightarrow$ $\Psi(T, X)$, the action reads $\int d T d X L[\Psi(T, X)]$ with a Lagrangian density,

$$
L[\Psi(T, X)]=\frac{1}{2} \Psi_{T T}^{2}-\Psi_{T}^{3}-\frac{1}{2} \Psi_{T} \Psi_{X},
$$

which contains higher-order $T$-time derivatives. The corresponding equation of motion,

$$
u_{T T T}+6 u u_{T}+u_{X}=0,
$$

is of third order in the $T$-time derivative of $u=\Psi_{T}$. As we will see below, higher-order $T$-derivatives in Eq. (4.6) bring about exponential instabilities when considering the evolution in the $T$ (i.e., $x$ ) direction.

In order to express our results on the $\mathrm{KdV}$ and modified $\mathrm{KdV}$ equations in a more transparent way, we will not henceforth use the notation $T$ for $x$ and $X$ for $t$. We will simply consider a nonstandard Cauchy problem for Eq. (4.3) [and its modified version, Eq. (4.10), considered below] written in a conventional way. Namely, instead of setting the initial value of $u(t, x)$ at ${ }^{9} t=0$ [i.e., giving one function of $x, v(x)$, with the condition $u(0, x)=v(x)$ ], we shall now pose a "rotated" Cauchy problem on the $x=0$ axis. However, as Eq. (4.3) features the third $x$ derivative of $u$, we must now give as Cauchy data three independent functions of $t, u_{0}(t), u_{1}(t)$, and $u_{2}(t)$, with the three conditions,

$u(t, 0)=u_{0}(t), \quad u_{x}(t, 0)=u_{1}(t), \quad u_{x x}(t, 0)=u_{2}(t)$.

\footnotetext{
${ }^{8}$ As usual, we denote the partial derivatives of $u(t, x)$ by subscripts; e.g., $u_{x} \equiv \partial u / \partial x$.

${ }^{9} \mathrm{As}$ the $\mathrm{KdV}$ equation is invariant under $t$ translations (and $x$ translations), one can fix the initial- $t$ Cauchy hypersurface at $t=$ 0 (and the initial- $x$ Cauchy hypersurface at $x=0$ ).
}

Note that this means that we are now considering more general solutions of the $\mathrm{KdV}$ equation. Indeed, any solution of the ordinary Cauchy problem [determined by one function $v(x)=u(0, x)$ ] will evolve in $t$ (in both directions, $t>0$ and $t<0$ ) and will induce on the $x=0$ axis some values for the three functions $u(t, 0)$, $u_{x}(t, 0)$, and $u_{x x}(t, 0)$, which are not functionally independent because they are all determined by the single function $v(x)$.

The ordinary ( $t$ direction) Cauchy problem for the KdV equation (4.3) has been shown to be globally well-posed when the single Cauchy datum $v(x)$ belongs to suitable functional spaces, namely $H^{s}$ Sobolev spaces with $s \geq 1$ (see [25] and references therein). However, the fact that the $\mathrm{KdV}$ equation has good dynamic behavior when evolved in the $t$ direction does not mean that the same is true for its evolution in the $x$ direction.

Indeed, we will explicitly see below that the $x$ evolution gives rise [when considering the linearized approximation of (4.3)] to exponentially growing modes (which are absent when considering the $t$ evolution). These exponentially growing modes amplify any small-scale structure present in the $x$-evolution Cauchy data $u_{0}(t), u_{1}(t)$, and $u_{2}(t)$ and are not tamed by the existence of the infinitely many conserved fluxes $\mathcal{F}_{n}$ defined in Eq. (4.2), because their integrands $J_{n}^{x}$ are not positive definite.

Worse, Eq. (4.3) admits approximate (as well as exact) solutions which blow up along a line located at some finite distance in the $x$ direction. Indeed, if one looks for power-law singularities of $u(t, x)$ of the general form $u(t, x) \approx C(t)\left[x-x_{0}(t)\right]^{\alpha}$, it is easily seen that they must necessarily be of the form,

$$
u(t, x)_{\mathrm{blowup}}^{\mathrm{KdV}} \approx-2\left[x-x_{0}(t)\right]^{-2},
$$

with an arbitrary possible blowup line $x=x_{0}(t)$. A specific example of such a blowup solution is the following exact $t$-independent solution of the $\mathrm{KdV}$ equation:

$$
u(t, x)=-\frac{2}{(x-c)^{2}},
$$

where $c$ is any constant. The function (4.9) has smooth Cauchy data on the $x=0$ axis, and blows up on the line $x=c$. We also performed some numerical simulations (with periodic Cauchy data given at $x=0$ ) and found that many such initial data develop a singularity of the form (4.8) during their $x$-direction evolution. This seems to confirm the standard "Ostrogradsky-ghost" lore that the evolution of a higher-derivative model, such as (4.5) becomes singular during their evolution. And one cannot expect that the quantum problem for the system (4.5) would be benign. 
However, the situation appears to be much better for the modified $^{10} \mathrm{KdV}$ equation,

$$
u_{x x x}+12 \kappa u^{2} u_{x}+u_{t}=0 .
$$

This equation admits an infinite number of integrals of motion, as the ordinary $\mathrm{KdV}$ equation does. The first three local conservation laws are

$$
\begin{gathered}
\partial_{t} u=-\partial_{x}\left(u_{x x}+4 \kappa u^{3}\right), \\
\partial_{t} u^{2}=-2 \partial_{x}\left[3 \kappa u^{4}+u u_{x x}-\frac{1}{2} u_{x}^{2}\right], \\
\partial_{t}\left(\kappa u^{4}-\frac{1}{2} u_{x}^{2}\right)=\partial_{x}\left[\kappa u_{x}\left(12 u^{2} u_{x}+u_{x x x}\right)\right. \\
\left.-\frac{1}{2} u_{x x}^{2}-4 \kappa u^{3} u_{x x}-8 \kappa^{2} u^{6}\right] .
\end{gathered}
$$

We will argue in the following that, in contrast to Eq. (4.3), the Eq. (4.10) does not involve a blowup.

Let us first briefly discuss the linearized KdV equation,

$$
u_{x x x}+u_{t}=0,
$$

which describes the fluctuations around the trivial solution $u(t, x)=0$ of the usual $\mathrm{KdV}$ equation (4.3), as well as of all its modified versions with the nonlinear term $n(n-1) \kappa u^{(n-2)} u_{x}, n \geq 3$.

Let us emphasize the drastic difference between the $t$-evolution Cauchy problem and the $x$-evolution Cauchy problem of the linearized equation (4.14).

Equation (4.14) can be solved by decomposing the solution $u(t, x)$ in plane waves $e^{\mathrm{i}(\omega t+k x)}$. This yields the dispersion law,

$$
\omega=k^{3} .
$$

If one poses the usual Cauchy problem with some Fouriertransformable initial data,

$$
u(0, x)=v(x) \equiv \int \frac{d k}{2 \pi} v(k) e^{i k x},
$$

the $t$ evolution of the initial data $v(x)$ yields the solution (valid for both signs of $t$ ),

\footnotetext{
${ }^{10}$ Modified KdV equations are generally defined by replacing the nonlinear $\mathrm{KdV}$ term $6 u u_{x}$ by $n(n-1) \kappa u^{(n-2)} u_{x}$. The usual $\mathrm{KdV}$ equation is the case $n=3$. In this case, a rescaling of the variables can set the coefficient $\kappa$ to 1 . Here, we consider the next modified $\mathrm{KdV}$ equation for $n=4$. In that case, a rescaling of the variables can set the coefficient $\kappa$ either to 1 or -1 ; these two cases having different physical properties - the so-called focusing and defocusing cases.
}

$$
u(t, x)=\int \frac{d k}{2 \pi} v(k) e^{\mathrm{i}\left(k^{3} t+k x\right)} .
$$

The important point here is that $u(t, x)$ is obtained from $v(k)$ by a purely oscillatory complex kernel $e^{\mathrm{i}\left(k^{3} t+k x\right)}$ of unit modulus. It has been shown that this oscillatory kernel has smoothing properties (see, e.g., [25]). This allows one to take the initial data in low- $s$ Sobolev spaces $H^{s}$ (describing pretty rough initial data) [25].

On the other hand, if one considers the $x$-evolution Cauchy problem, one starts from three independent functions of $t$ along the $x=0$ axis: $u(t, 0)=u_{0}(t)$, $u_{x}(t, 0)=u_{1}(t)$, and $u_{x x}(t, 0)=u_{2}(t)$; see Eq. (4.7). Assuming that the three Cauchy data $u_{a}(t), a=0,1,2$, are Fourier-transformable, we can represent them as

$$
u_{a}(t) \equiv \int \frac{d \omega}{2 \pi} u_{a}(\omega) e^{\mathrm{i} \omega t}
$$

The three Cauchy data determine a unique solution which, when decomposed in plane waves, satisfies the same dispersion law (4.15) as before. However, the dispersion law (4.15) must now be solved for $k$ in terms of $\omega$. As it is a cubic equation in $k$, it has three different roots, namely,

$$
k_{a}(\omega)=\omega^{\frac{1}{3}} j^{a},
$$

where $\omega^{\frac{1}{3}}$ denotes the unique real cubic root of $\omega$ and where $j^{a}=1, j, j^{2}$ (with $j \equiv e^{\frac{2 \pi i}{3}}=-\frac{1}{2}+\mathrm{i} \frac{\sqrt{3}}{2}$ ) are the three complex roots of unity. This yields a solution for $u(t, x)$ of the form,

$$
u(t, x)=\sum_{a=0,1,2} \int \frac{d \omega}{2 \pi} v_{a}(\omega) e^{\mathrm{i}\left(\omega t+k_{a} x\right)},
$$

where the three coefficients $v_{a}(\omega)$ are (uniquely) determined by the three initial conditions at $x=0$, namely by the following system of three linear equations ${ }^{11}$ :

$$
\begin{aligned}
& u_{0}(\omega)=\sum_{a=0,1,2} v_{a}(\omega), \\
& u_{1}(\omega)=\mathrm{i} \sum_{a=0,1,2} k_{a}(\omega) v_{a}(\omega), \\
& u_{2}(\omega)=-\sum_{a=0,1,2} k_{a}^{2}(\omega) v_{a}(\omega) .
\end{aligned}
$$

The point of this exercise was to exhibit the fact that, when considering the $x$ evolution with arbitrary Cauchy data $u_{0}(t), u_{1}(t), u_{2}(t)$, the solution involves exponentially growing modes in the $x$ direction, linked to the fact that $\mathrm{i} k_{1}=\mathrm{i} \omega^{\frac{1}{3}} j$ and $\mathrm{i} k_{2}=\mathrm{i} \omega^{\frac{1}{3}} j^{2}$ have real parts $\pm \frac{\sqrt{3}}{2} \omega^{\frac{1}{3}}$

\footnotetext{
${ }^{11}$ The determinant of this system is $-3 \sqrt{3} \omega$.
} 
(this holds for both signs of $x$ ). This instability is associated with the presence of higher time derivatives. ${ }^{12}$

From the mathematical point of view, this means that high-frequency (HF) wiggles, in the sense of high $\omega$, in the initial Cauchy data (at $x=0$ ) will be amplified, on both sides of the $x$ axis, by the exponentially growing factor $e^{\frac{\sqrt{3}}{2}|\omega|^{\frac{1}{3}}|x|}$. This indicates that the Cauchy problem will be well-posed only if one takes initial data whose Fourier transforms $v_{a}(\omega)$ decrease sufficiently fast as $|\omega| \rightarrow+\infty$. As a minimum condition for a local existence theorem, one should require the Fourier transforms $v_{a}(\omega)$ to decrease like $e^{-c|\omega|^{\frac{1}{3}}}$ for some positive constant $c$. This essentially defines the $s=3$ Gevrey class of functions on $\mathbb{R}$ (see, e.g., [26]). Note that such a regularity condition is stronger than infinite differentiability, but weaker than analyticity (which corresponds to a decrease of the Fourier transforms of the type $e^{-c|\omega|}$, i.e., to the $s=1$ Gevrey class).

We therefore expect that it will be mathematically possible to prove (at least for sufficiently weak Cauchy data) a local existence theorem for solutions of the $x$-evolution of the modified KdV equation (4.10) in suitable Gevrey classes (say, with $1<s \leq 3$ ). ${ }^{13}$

We wish to further argue that the special nonlinearity of Eq. (4.10), when taking $\kappa>0$, is likely to allow sufficiently smooth $x=0$ Cauchy data to define global solutions, extending to arbitrary large values of $|x|$. Our main argument for believing that the local $x$ evolution of Eq. (4.10) can be extended to large values of $|x|$ is the absence of blowup solutions. Indeed, let us look for power-law singularities of $u(t, x)$ of the general form,

$$
u(t, x) \approx \beta(t)\left[x-x_{0}(t)\right]^{\alpha},
$$

with $\alpha<0$. Inserting such an asymptotic behavior in Eq. (4.10), it is easily seen that the $t$-derivative term $u_{t}$ is necessarily subdominant with respect to the $x$-derivative terms. Therefore, blowup solutions must, in lowest approximation, solve the truncated equation,

$$
0=u_{x x x}+12 \kappa u^{2} u_{x}=\frac{\partial}{\partial x}\left(u_{x x}+4 \kappa u^{3}\right) .
$$

This equation is a third-order dynamical equation for the $x$ evolution which admits the "constant of motion,"

\footnotetext{
${ }^{12}$ We hasten to comment, however, that higher derivatives do not necessarily entail such an instability. For example, it does not show up in the equations of motion for the Pais-Uhlenbeck system (1.1).

${ }^{13}$ Nader Masmoudi confirmed (private communication to TD) that it was likely that taking Cauchy data in the $s=3$ Gevrey class would suffice for local existence of the solution of the $x$ evolution. This would still leave open the issue of finding adequate function spaces for global existence.
}

$$
u_{x x}+4 \kappa u^{3}=C
$$

In turn, the latter equation can be rewritten as $u_{x x}=-\frac{d V(u)}{d u}$, i.e., as the Newtonian equation of motion in $x$, playing the role of time, for a particle with position $u$ in the following potential:

$$
V(u)=\kappa u^{4}-C u .
$$

When $\kappa>0$ (the focusing case), this potential grows for large values of $|u|$, and therefore prevents the existence of blowup solutions. ${ }^{14}$ Technically, if we look for blowup solutions of the type (4.22), one finds that the blowup exponent $\alpha$ must be equal to $\alpha=-1$ and that the coefficient $\beta$ must satisfy the cubic equation $\beta\left(2 \kappa \beta^{2}+1\right)=0$. When $\kappa<0$ (the defocusing case), there exist possible nontrivial (real) blowup solutions with $\beta= \pm(-2 \kappa)^{-\frac{1}{2}}$. [These solutions correspond to the "fall" of the $u$ particle down the unstable quartic potential $-|\kappa| u^{4}-C u$.] By contrast, when $\kappa>0$, the only real solution of the cubic equation of $\beta$ is $\beta=0$. In other words, there exists no real blowup solutions of the type indicated in Eq. (4.22). ${ }^{15}$

A different (though related) analytic argument indicating the absence of real blowup solutions comes from the analysis of the scaling properties of the modified $\mathrm{KdV}$ equation. It is easily seen that Eq. (4.10) is invariant under the rescalings $u=\lambda_{u} \bar{u}, x=\lambda_{x} \bar{x}, t=\lambda_{t} \bar{t}$ if

$$
\lambda_{t}=\lambda_{x}^{3} ; \quad \lambda_{u}=\lambda_{x}^{-1} .
$$

The quantities $x u$ and $\frac{x}{t^{1 / 3}}$ are invariant under these rescalings. Using also the space and time translational invariance of the modified KdV equation, we can look for scaling solutions of the type,

$$
u(t, x)=\frac{\alpha}{\left[3\left(t-t_{0}\right)\right]^{1 / 3}} w(z),
$$

where

$$
z \equiv \frac{x-x_{0}}{\left[3\left(t-t_{0}\right)\right]^{1 / 3}}
$$

Inserting the ansatz (4.27) in Eq. (4.10) and choosing the normalization constant $\alpha=\frac{1}{\sqrt{2}}$, it is easily checked that the function $w(z)$ must satisfy the equation

\footnotetext{
${ }^{14} \mathrm{By}$ contrast, for the ordinary $\mathrm{KdV}$, one obtains a nonconfining cubic potential, and the corresponding equations of motion admit the singular run-away solutions (4.9). General modified $\mathrm{KdV}$ equations with the nonlinearity $n(n-1) \kappa u^{(n-2)} u_{x}$ give rise to a potential $V(u)=\kappa u^{n}-C u$, which is confining if $n$ is even and $\kappa>0$.

${ }^{15}$ We are aware that the ansatz (4.22) might be too restrictive. It was shown in Ref. [27] that, in the formally confining case $n=6$ with $\kappa>0$, a blowup can occur in the $t$ evolution for data close to the corresponding soliton.
} 
$0=w^{\prime \prime \prime}+\left(6 \kappa w^{2}-z\right) w^{\prime}-w=\frac{d}{d z}\left[w^{\prime \prime}+2 \kappa w^{3}-z w\right]$.

Denoting as $C$ the constant value of the bracket in the last right-hand side, we conclude that $w(z)$ satisfies a secondorder equation of the type

$$
w^{\prime \prime}=-2 \kappa w^{3}+z w+C .
$$

This is a Painlevé II equation [28]. In general, Painlevé equations have (moving) pole singularities. And indeed, a local analysis of Eq. (4.30) (keeping the leading-order terms $\left.{ }^{16} w^{\prime \prime} \approx-2 \kappa w^{3}\right)$ shows that (4.30) admits simple poles, $w(z)=R /\left(z-z_{0}\right)$, as local singularities if the residue $R$ satisfies the equation $\kappa R^{2}=-1$. When $\kappa<0$, the residue $R$ will be real, so that real solutions of Painlevé II can have (and generally do have) poles on the real $z$ axis. The existence of a real simple pole at $z=z_{0}$ would then correspond to a singular (blowup) behavior of $u(t, x)$ of the form $u(t, x) \propto\left[x-x_{0}-z_{0}\left[3\left(t-t_{0}\right)\right]^{1 / 3}\right]^{-1}$. However, when $\kappa>0$, real solutions cannot have real poles. This excludes the existence of (real) singular scaling solutions.

Another way to understand why the negative- $\kappa$ modified $\mathrm{KdV}$ equation has blowup solutions is to use its relation with the usual $\mathrm{KdV}$ equation (4.3) (which admits real blowup solutions, as we emphasized above). Indeed, it is well-known that the Miura transformation,

$$
u=-\left(2 v^{2}+\sqrt{2} v_{x}\right),
$$

transforms the ordinary $\mathrm{KdV}$ equation (4.3) for $u(t, x)$ into the equation,

$$
v_{t}-12 v^{2} v_{x}+v_{x x x}=0,
$$

which coincides with the modified $\mathrm{KdV}$ equation (4.10) for $v(t, x)$, with $\kappa=-1$.

To confirm our conjecture that the $x$ evolution of sufficiently smooth Cauchy data (4.7) stays bounded when evolved with the modified $\mathrm{KdV}$ equation with $\kappa>0$, we have performed some numerical simulations (done with Mathematica) for the case $\kappa=+1$. To simplify the numerical analysis, we imposed [as is allowed by Eq. (4.10)] periodicity along the $t$ direction Using scaling invariance, we can assume $2 \pi$ periodicity,

$$
u(t+2 \pi, x)=u(t, x) .
$$

In order to study the effect of the nonlinear term $12 u^{2} u_{x}$, we used $t$-periodic Cauchy data for which the nonlinear term is initially (i.e., at $x=0$ ) larger than the linear term $u_{t}$. In particular, we used the Cauchy data,

\footnotetext{
${ }^{16}$ Note in passing that this leading-order equation describe the dynamics of a particle in the potential $V(w)=\frac{1}{2} \kappa w^{4}$.
}

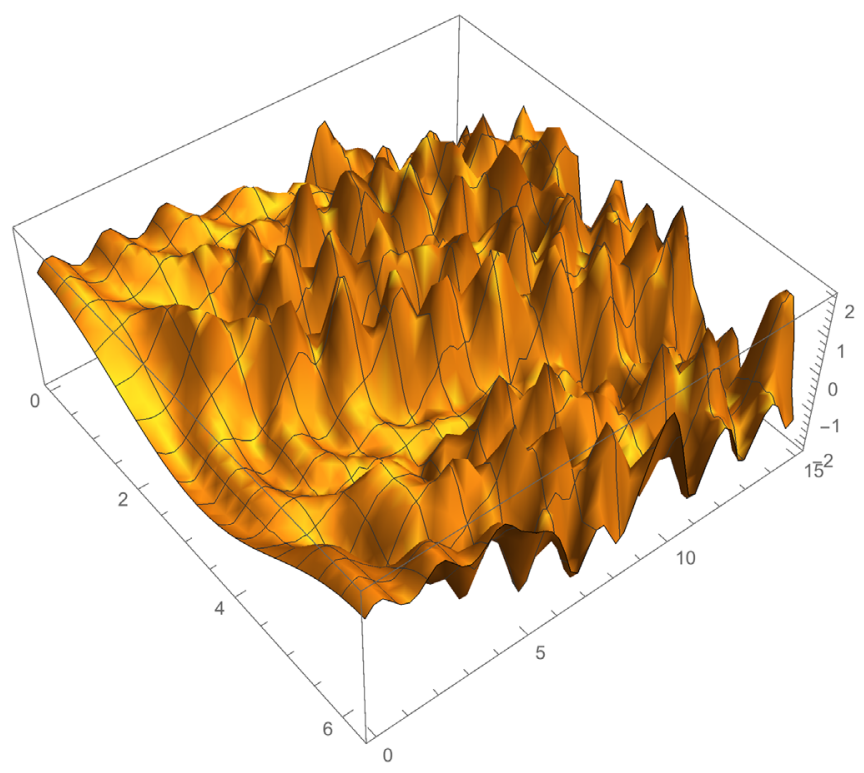

FIG. 2. Solution $u(t, x)$ of the $\kappa=+1$ modified KdV equation (4.10) corresponding to the $t$ periodicity (4.33) and the $x=0$ Cauchy data (4.34).

$$
u(t, 0)=\cos t, \quad u_{x}(t, 0)=\cos t, \quad u_{x x}(t, 0)=0 .
$$

We first checked that the use of such Cauchy data for the modified $\mathrm{KdV}$ equation with $\kappa=-1$ was leading quite fast (namely at $x=0.887717$ ) to a singularity. By contrast, our numerical simulations of the $x$ evolution of the $\kappa=+1$ modified $\mathrm{KdV}$ equation showed that $u(t, x)$ stayed bounded for all the values of $x$ that we explored. This is illustrated in Fig. 2, which displays the solution $u(t, x)$ generated by the Cauchy data (4.34) in the domain $0 \leq t \leq 2 \pi, 0 \leq x \leq 15$.

Figure 2 illustrates the benign nature of the ghostful $x$ dynamics of the modified KdV equation in the positive- $\kappa$ case. The presence (when neglecting the term $u_{t}$ ) of an approximate $x$ dynamics governed by the confining potential (4.25) reflects itself in the oscillations in the $x$ evolution of $u(t, x)$, i.e., in the "stormy-sea" aspect of $u(t, x)$ in the $x>5$ part of Fig. 2. Note that we have taken here analytic $\left(C^{\omega}\right)$ data, which generate (at least locally) an analytic solution. We leave to future work to clarify how less regular Cauchy data (e.g., taken in Gevrey classes, or suitable Sobolev-type spaces) would evolve under the $x$ evolution.

We expect generic Cauchy data (4.7) for the $x$ evolution to evolve into stormy-sea solutions similar to the one illustrated in Fig. 2. However, there will also exist special (measure-zero) Cauchy data that will evolve into much tamer solutions. Indeed, if we start with a smooth function of $x$, say $v(x)$, and use it as unique Cauchy datum at $t=0$ [namely, $u(t=0, x)=v(x)$ ], its $t$ evolution will define a smooth solution $u^{v \text { sol }}(t, x)$. The restriction to the $x=0$ axis of $u^{v \text { sol }}, u_{x}^{v \text { sol }}$, and $u_{x x}^{v \text { sol }}$ will then define Cauchy data for the $x$ evolution that generate the smooth solution $u^{v \text { sol }}(t, x)$. 
We have numerically checked this fact by using solitonic solutions of the modified $\mathrm{KdV}$ equation.

Let us recall that, like the ordinary $\mathrm{KdV}$ equation, the modified equation (4.10) admits solitonic solutions. Indeed, one can look for traveling-wave solutions $u(t, x)=$ $u(x+c t)$, moving with some celerity $c$, by inserting the ansatz $u(t, x)=u(\bar{x})$, with $\bar{x} \equiv x+c t$, in Eq. (4.10). It is easily seen that this yields the equation,

$$
\frac{\partial}{\partial \bar{x}}\left[c u+4 \kappa u^{3}+u_{\bar{x} \bar{x}}\right]=0 .
$$

Denoting as $C_{0}$ the constant quantity within the bracket, we then get the following second-order equation for the function $u(\bar{x})$ :

$$
u_{\bar{x} \bar{x}}=-\frac{d}{d u} \mathcal{V}(u),
$$

with a potential function $\mathcal{V}(u)$ now given by

$$
\mathcal{V}(u)=\kappa u^{4}+\frac{1}{2} c u^{2}-C_{0} u .
$$

We are again reduced to the dynamics of a particle moving in the confining quartic potential $\mathcal{V}(u)$, considered for some fixed $\kappa>0$ (say, $\kappa=+1$ ). The general solutions of this problem then depend on three parameters: the celerity $c$, the constant $C_{0}$, and the constant energy of the $\bar{x}$ dynamics,

$$
E=\frac{1}{2} u_{\bar{x}}^{2}+\mathcal{V}(u) .
$$

The usually considered solitonic solutions [such that $u(\bar{x})$ tend to zero when $\bar{x} \rightarrow \pm \infty]$ are obtained by taking $c<0$, $C_{0}=0$ and $E=0$. This gives a symmetric double-well potential: $\kappa u^{4}-\frac{1}{2}|c| u^{2}$. The zero-energy solution then describes a $u$ motion, which starts, at "time" $\bar{x}=-\infty$, at $u=0$ with zero "velocity" $u_{\bar{x}}$, glides down (say) to the right, reflects on the right wall of the double well and then turns back to end up again at $u=0$ when $\bar{x}=+\infty$. The explicit form of the corresponding solution is

$$
u(\bar{x})=\sqrt{\frac{|c|}{2 \kappa}} \frac{1}{\cosh (\sqrt{|c|} \bar{x})} .
$$

Here, we are interested in constructing periodic traveling-wave solutions satisfying $u(\bar{x}+\bar{T})=u(\bar{x})$ for some $\bar{T}$. Such solutions can be easily constructed by considering bounded mechanical motions in the potential $\mathcal{V}(u)$ having a nonzero energy. We have already given such oscillatory solutions in a (symmetric) quartic potential (i.e., for $C_{0}=0$ ) in Eq. (2.16) above. The corresponding periodic traveling-wave soliton is then of the form,

$$
u(\bar{x})=x_{0} \operatorname{cn}\left[\Omega\left(\bar{x}-\bar{x}_{0}\right), k\right],
$$

as simply obtained by using in Eqs. (2.13), (2.16), (2.17) the replacements

$$
x \rightarrow u, t \rightarrow \bar{x}, \omega^{2} \rightarrow c, \lambda \rightarrow 4 \kappa, N \rightarrow E .
$$

We have used the $t$-periodic Cauchy data defined by restricting $u(x+c t)$, and its first two $x$ derivatives, to $x=0$ to check the accuracy of our numerical simulations. The numerical solution generated from these Cauchy data agreed well with the analytical solution for values of $x$ of order $\bar{T}$. For larger values, they exhibited some numerical noise. The specific form of the numerical noise depended on the numerical scheme used. When using the same scheme as the one used to produce Fig. 2, the noise stayed at low frequencies. This gives us confidence that Fig. 2 yields a reasonably accurate picture of the benign nature of the $x$ evolution of smooth Cauchy data.

\section{DISCRETE NONLINEAR SYSTEMS WITH BENIGN GHOSTS}

In this final section, we define some higher-derivative dynamical models having only a finite number of degrees of freedom and exhibiting a benign behavior in their evolution. These models are constructed by discretizing the modified $\mathrm{KdV}$ equation in the $t$ direction, keeping continuous the $x$ evolution. [We recall that $x$ is our timelike evolution variable.] This will replace the partial differential equation (4.10) by a system of coupled ODEs with respect to $x$.

Similarly to the derivation of the ordinary KdV equation from the Lagrangian (4.4), the modified $\mathrm{KdV}$ equation (4.10) follows from the two-dimensional action $S=\int d t d x L[\psi(t, x)]$, with the field-theory Lagrangian density,

$$
L[\psi(t, x)]=\frac{1}{2} \psi_{x x}^{2}-\kappa \psi_{x}^{4}-\frac{1}{2} \psi_{x} \psi_{t} .
$$

After varying the action with respect to $\psi(t, x)$, one gets Eq. (4.10) by substituting $\psi_{x}(t, x) \rightarrow u(t, x)$.

We can then define a discretized version of the modified $\mathrm{KdV}$ dynamics by assuming that the variable $t$ takes only the discrete values $t=h, 2 h, \ldots, N h$, for some integer $N \geq 2$, and by replacing the continuous time derivative $\psi_{t}$ by a discrete (symmetric) time derivative $\frac{\psi(t+h, x)-\psi(t-h, x)}{2 h}$. This yields an action of the form $S_{N}=\int d x L_{N}$, where the Lagrangian $L_{N}$ is given by a sum of $N$ terms,

$$
\begin{aligned}
L_{N}= & \sum_{t=h}^{t=N h}\left[\frac{1}{2}\left[\psi_{x x}(t, x)\right]^{2}-\kappa\left[\psi_{x}(t, x)\right]^{4}\right. \\
& \left.-\frac{1}{2} \psi_{x}(t, x) \frac{\psi(t+h, x)-\psi(t-h, x)}{2 h}\right] .
\end{aligned}
$$


To define the model, we further need to specify boundary conditions. Namely, we need to define $\psi(0 h, x)$ and $\psi[(N+1) h, x]$, which enter the discrete $t$ derivative for $t=$ $h$ and $t=N h$, respectively. This can be done in two different ways: (i) we can use Dirichlet-type boundary conditions, namely $\psi(0 h, x)=0$ and $\psi[(N+1) h, x]=0$ or (ii) periodic boundary conditions, $\psi(0 h, x)=\psi(N h, x)$, and $\psi[(N+1) h, x]=\psi(h, x)$. The periodicity condition can only be imposed when $N \geq 3$.

We expect that taking larger and larger values of $N$ would allow one to simulate better and better the continuous theory (though the presence of chaos might make such a convergence nonuniform in $x$ ).

The simplest discretized model is obtained by choosing $N=2$ and taking Dirichlet-type boundary conditions. This model has only two dynamical variables: $\psi(x) \equiv \psi(h, x)$ and $\chi(x) \equiv \psi(2 h, x)$. We recall that $x$ is playing the role of time. The Lagrangian $L_{2} \equiv L_{N=2}$ reads

$$
L_{2}=\frac{1}{2} \psi_{x x}^{2}+\frac{1}{2} \chi_{x x}^{2}-\kappa \psi_{x}^{4}-\kappa \chi_{x}^{4}-\frac{1}{4 h} \psi_{x} \chi+\frac{1}{4 h} \chi_{x} \psi .
$$

Adding a total $x$ derivative, the last two terms can be traded for $+\frac{1}{2 h} \chi_{x} \psi$. Using suitable rescalings, we can set $\kappa=1$ and $h=\frac{1}{2}$. For simplicity, we will use these values in the following.

Defining the two new dynamical variables $a(x) \equiv \psi_{x}$, $b(x) \equiv \chi_{x}$, the equations of motion derived from the Lagrangian (5.3) read

$$
\begin{aligned}
& a_{x x x}+12 a^{2} a_{x}+b=0, \\
& b_{x x x}+12 b^{2} b_{x}-a=0 .
\end{aligned}
$$

This is a system of two coupled higher-order evolution equations. The important point is that, while we had technical difficulties in numerically simulating in a stable manner the $x$ evolution of the (periodic) modified $\mathrm{KdV}$ equation (4.10), we could easily perform long-term integrations of the coupled system (5.4) up to $x=10000$ and more in a numerically stable manner. And though the conserved energy of the system, namely,

$$
\begin{aligned}
E & =\frac{1}{2}\left(\psi_{x x}^{2}+\chi_{x x}^{2}\right)-3\left(\psi_{x}^{4}+\chi_{x}^{4}\right)-\psi_{x} \psi_{x x x}-\chi_{x} \chi_{x x x} \\
& =\frac{1}{2}\left(a_{x}^{2}+b_{x}^{2}\right)-3\left(a^{4}+b^{4}\right)-a a_{x x}-b b_{x x}
\end{aligned}
$$

[which is a discrete version of the conserved current on the right-hand side of Eq. (4.12)], is not positive-definite and can take arbitrarily positive or negative values, our numerical simulations indicate that the classical motions have a benign behavior, without any blowup. In other words, the simple discrete model $L_{2}$, Eq. (5.3), provides a nontrivial example of an interacting higher-derivative system with benign ghosts.
One can also write the corresponding Ostrogradsky Hamiltonian. It reads

$$
H=\frac{1}{2}\left(P_{\psi}^{2}+P_{\chi}^{2}\right)+p_{\psi} \psi_{x}+p_{\chi} \chi_{x}+\psi_{x}^{4}+\chi_{x}^{4}-\psi \chi_{x},
$$

where $\psi, \psi_{x}, \chi, \chi_{x}$ should all be treated as independent variables with corresponding canonical momenta $p_{\psi}, P_{\psi}$, $p_{\chi}$ and $P_{\chi}$, respectively. The Hamilton equations of motion following from (5.6) coincide with (5.4).

For completeness, let us also write the periodic, discrete dynamics defined by $L_{N}$, Eq. (5.2), with $N \geq 3$. Setting as before $\kappa=1$ and $h=\frac{1}{2}$ and defining the $N x$-evolving discrete variables $a^{k}(x) \equiv \psi(k h, x)_{x}$, with $k=1, \ldots, N$, and the periodicity conditions, $a^{0} \equiv a^{N}$ and $a^{N+1} \equiv a^{1}$, the equations of motion following from Eq. (5.2) read

$$
a_{x x x}^{k}+12\left(a^{k}\right)^{2} a_{x}^{k}+\left(a^{k+1}-a^{k-1}\right)=0 .
$$

The conserved energy is

$$
E=\sum_{k=1}^{N}\left[\frac{1}{2}\left(a_{x}^{k}\right)^{2}-3\left(a^{k}\right)^{4}-a^{k} a_{x x}^{k}\right] .
$$

The periodic systems with $N \geq 3$ enjoy also a second integral of motion (linked to the periodicity in $t_{k}=k h$ ),

$$
Q=\sum_{k=1}^{N}\left[a_{x x}^{k}+4\left(a^{k}\right)^{3}\right] .
$$

In the continuous theory, this follows from integrating over the periodic variable $t$ the current in the right-hand side of Eq. (4.11). By contrast, the currents in the higher conservation laws of the modified $\mathrm{KdV}$ equation, starting with Eq. (4.13), do not translate into integrals of motion of the discrete systems.

The $N$ th periodic discrete system has only two integrals of motion, Eqs. (5.8), (5.9), and $2 N$ pairs of phase space variables. [For $N=2$, one had only one integral of motion for four pairs of phase space variables.] The systems (5.2) are thus not integrable, and their trajectories are expected to exhibit a chaotic behavior. Our numerical simulations did confirm this expectation.

The numerical confirmations of the benign nature of the discrete systems up to rather large values of $x$ can be considered as a further argument in favor of the conjecture that the continuous modified KdV system (4.10) is also benign.

\section{CONCLUSIONS}

We presented several nontrivial examples of higherderivative systems including ghosts, but where the ghosts are of benign nature; i.e., they do not lead to a blowup in the classical case (and hence will not give rise to unitarity 
violation in the quantum case). The most interesting example is the two-dimensional modified $\mathrm{KdV}$ system (5.1) (with $\kappa>0$ ), viewed as a higher-derivative evolution in the $x$ variable. We presented several arguments strongly indicating that this system does not involve any blowup during its $x$ evolution. Mathematically proving that this is the case (for smooth enough data) is a challenge for the future.

In the last section, we presented mechanical systems, with a finite number of degrees of freedom, which are $t$-discretized avatars of the modified KdV system (5.1). These systems are of interest on their own, notably because they provide a set of nontrivial interacting higher derivative systems with benign ghosts. Such systems were not known before.

\section{ACKNOWLEDGMENTS}

We are indebted to Piotr Chrusciel, Alberto De Sole, Cedric Deffayet, Victor Kac, Olaf Lechtenfeld, Nader Masmoudi, Frank Merle, and Laure Saint-Raymond for informative discussions. A.S. aknowledges the warm hospitality of IHES.
[1] M. Ostrogradsky, Mémoire sur les équations différentielles relatives au problème des isopérimètres, Mem. Acad. St. Petersbourg VI 4, 385 (1850).

[2] A. Smilga, Classical and quantum dynamics of higherderivative systems, Int. J. Mod. Phys. A 32, 1730025 (2017).

[3] A. Pais and G. E. Uhlenbeck, On field theories with nonlocalized action, Phys. Rev. 79, 145 (1950).

[4] E. Pagani, G. Tecchiolli, and S. Zerbini, On the problem of stability for higher order derivatives: Lagrangian systems, Lett. Math. Phys. 14, 311 (1987).

[5] P. D. Mannheim and A. Davidson, Dirac quantization of Pais-Uhlenbeck fourth order oscillator, Phys. Rev. A 71, 042110 (2005).

[6] K. Bolonek and P. Kosinski, Comments on "Dirac quantization of the Pais-Uhlenbeck fourth order oscillator", arXiv:quant-ph/0612009.

[7] A. V. Smilga, Comments on the dynamics of the PaisUhlenbeck oscillator, SIGMA 5, 017 (2009).

[8] A. V. Smilga, Benign vs malicious ghosts in higherderivative theories, Nucl. Phys. B706, 598 (2005).

[9] S. N. Carroll, M. Hoffman, and M. Trodden, Can the dark energy equation-of-state parameter $w$ be less than -1 ?, Phys. Rev. D 68, 023509 (2003).

[10] K. M. Case, Singular potentials, Phys. Rev. 80, 797 (1950); K. Meetz, Singular potentials in non-relativistic quantum mechanics, Nuovo Cimento 34, 690 (1964); A. M. Perelomov and V.S. Popov, Collapse onto scattering center in quantum mechanics, Theor. Math. Phys. 4, 664 (1970).

[11] D. Robert and A. V. Smilga, Supersymmetry vs ghosts, J. Math. Phys. (N.Y.) 49, 042104 (2008).

[12] I. B. Ilhan and A. Kovner, Some comments on ghosts and unitarity: The Pais-Uhlenbeck oscillator revisited, Phys. Rev. D 88, 044045 (2013).

[13] M. Pavšič, Stable self-interacting Pais-Uhlenbeck oscillator, Mod. Phys. Lett. A 28, 1350165 (2013).

[14] A. V. Smilga, Supersymmetric field theory with benign ghosts, J. Phys. A 47, 052001 (2014).

[15] C. Deffayet, S. Mukohyama, and A. Vikman, Ghosts Without Runaway, Phys. Rev. Lett. 128, 041301 (2022).
[16] J. Ginibre and G. Velo, The global Cauchy problem for the non linear Klein-Gordon equation-II, Ann. Inst. Henri Poincaré, C 6, 15 (1989).

[17] D. Christodoulou and S. Klainerman, The Global Nonlinear Stability of the Minkowski Space (Princeton University Press, Princeton, NJ, 1993).

[18] H. Lindblad and I. Rodnianski, The global stability of the Minkowski space-time in harmonic gauge, Ann. Math. 171, 1401 (2010).

[19] L. Bieri, An extension of the stability theorem of the Minkowski space in general relativity, J. Diff. Geom. 86, 17 (2010).

[20] Y. Choquet-Bruhat, P. T. Chrusciel, and J. Loizelet, Global solutions of the Einstein-Maxwell equations in higher dimensions, Classical Quantum Gravity 23, 7383 (2006).

[21] J. Loizelet, Solutions globales des équations d'EinsteinMaxwell, Ann. Fac. Sci. Toulouse (Mathématiques), 18, 495 (2009).

[22] S. W. Hawking and G. F. R. Ellis, The Large Scale Structure of Space-Time (Cambridge University Press, Cambridge, England, 1973).

[23] C. W. Misner, Taub-NUT space as a counterexample to almost anything, in Relativity Theory and Astrophysics I: Relativity and Cosmology, edited by J. Ehlers, Lectures in Applied Mathematics, Vol. 8 (American Mathematical Society, Providence, RI, 1967), pp. 160-169.

[24] A. V. Smilga, On exactly solvable ghost-ridden systems, Phys. Lett. A 389, 127104 (2021).

[25] C. E. Kenig, G. Ponce, and L. Vega, Well-posedness of the initial value problem for the Korteweg-de Vries equation, J. Am. Math. Soc. 4, 323 (1991).

[26] L. Rodino, Linear Partial Differential Operators in Gevrey Spaces (World Scientific, Singapore, 1993), p. 264.

[27] Y. Martel, F. Merle, and P. Raphaël, Blow up for the critical generalized Korteweg-de Vries equation. I: Dynamics near the soliton, Acta. Math. 212, 59 (2014).

[28] See e.g., P. A. Clarkson, Painlevé equations-nonlinear special functions, J. Comput. Appl. Math. 153, 127 (2003). 